\title{
The Functional Neuroanatomy of Temporal Discrimination
}

\author{
Maria A. Pastor, ${ }^{1,2}$ Brian L. Day, ${ }^{2}$ Emiliano Macaluso, ${ }^{3,5}$ Karl J. Friston, ${ }^{4}$ and Richard S. J. Frackowiak ${ }^{4,5}$ \\ ${ }^{1}$ Department of Neurology, University of Navarre School of Medicine, Clinica Universitaria, 31080 Pamplona, Spain, ${ }^{2}$ Sobell Department of Movement \\ Neuroscience, Institute of Neurology, University College of London, ${ }^{3}$ Institute of Cognitive Neuroscience, University College of London, and ${ }^{4}$ Wellcome \\ Department of Imaging Neuroscience, Institute of Neurology, University College of London, London WC1N 3BG, United Kingdom, and 5Neuroimaging \\ Laboratory, Fondazione Santa Lucia, Instituto di Ricovero e Cura a Carattere Scientifico, Rome, Italy
}

Two identical stimuli, such as a pair of electrical shocks to the skin, are readily perceived as two separate events in time provided the interval between them is sufficiently long. However, as they are presented progressively closer together, there comes a point when the two separate stimuli are perceived as a single stimulus. Damage to posterior parietal cortex, peri-supplementary motor area (peri-SMA), and basal ganglia can disturb this form of temporal discrimination. Our aim was to establish, in healthy subjects, the brain areas that are involved in this process. During functional magnetic resonance imaging scanning, paired electrical pulses, separated by variable interstimulus intervals $(5-110 \mathrm{msec})$, were delivered to different sites on one forearm $(8-64 \mathrm{~mm}$ from the midline). Subjects were required to simply detect the stimulus (control task) or to identify a stimulus property. For temporal discrimination (TD), subjects reported whether they felt one or two stimuli. For spatial discrimination, they reported whether the stimuli were located on the right or left side of the forearm. Subjects reported their choice by pressing a button with the opposite hand. Our results showed that discrimination, as opposed to simply detection, activated several brain areas. Most were common to both discrimination tasks. These included regions of prefrontal cortex, right postcentral gyrus and inferior parietal lobule, basal ganglia, and cerebellum. However, activation of pre-SMA and anterior cingulate was found to be specific to the TD task. This suggests that these two frontal regions may play a role in the temporal processing of somatosensory events.

Key words: tactile temporal discrimination; simultaneity-succession discrimination; pre-SMA; basal ganglia; cerebellum; prefrontal cortex; event-related fMRI

\section{Introduction}

In the somatosensory domain, most healthy subjects are able to perceive two stimuli as being sequential when the inter-stimulus interval (ISI) exceeds $\sim 30-50 \mathrm{msec}$. Damage to specific regions of the brain can compromise performance of this task. For instance, the inter-stimulus interval required for the discrimination is increased in patients with focal lesions that do not cause overt sensory impairment, such as in the supplementary motor area (SMA), posterior parietal cortex, and basal ganglia (Lacruz et al., 1991). Similarly, raised thresholds have been found in patients with basal ganglia disorders, including Parkinson's disease (Artieda et al., 1992) and dystonia (Sanger et al., 2001; Tinazzi et al., 2002). In Parkinson's disease, discrimination threshold is related to the severity of the disease and is reduced by dopaminergic treatment. In these patients, the raised threshold is unlikely to be attributable to a failure of sensory transmission, because median nerve and cortical somatosensory evoked potential recovery curves after double electrical stimulation are normal (Artieda et

Received Sept. 13, 2003; revised Jan. 10, 2004; accepted Jan. 13, 2004.

This work was funded by the Wellcome Trust. M.A.P. was supported by Grant EX2001 46214114 from the Spanish Ministerio de Educacion y Cultura-Fulbright. We thank R. Bedlington and P. Allen for technical support and Prof. Jose Masdeu for his valuable suggestions on this manuscript.

Correspondence should be addressed to Dr. Maria A. Pastor, Department of Neurology, University of Navarre School of Medicine, Clinica Universitaria, 31080 Pamplona, Spain. E-mail: mapastor@unav.es.

DOI:10.1523/JNEUROSCI.4210-03.2004

Copyright $\odot 2004$ Society for Neuroscience $\quad$ 0270-6474/04/242585-07\$15.00/0 al., 1992). The perception of two stimuli as being either sequential or temporally coincident is thought to involve brain processes concerned with timing function (Nichelli, 1993). Therefore, raised discrimination thresholds may represent a deficit in the temporal processing of normal sensory input.

Except for postlesional studies, the location of brain areas involved in this temporal discrimination (TD) has not been explored. To characterize the functional anatomy, we used functional magnetic resonance imaging (fMRI) while subjects performed a TD task. A comparison of the fMRI activation pattern during discrimination with that during simple detection (using identical stimuli and motor responses) should disclose brain regions involved in this form of TD. However, within this net activation pattern, it would not be possible to distinguish nonspecific processes, such as those associated with the attentional demands of the task, from the specific processes involved in TD. Therefore, we used a third task in which subjects were required to perform an equally demanding discrimination task but one directed toward a spatial attribute of the stimulus [spatial discrimination (SD)].

Because all three tasks used identical stimulus sets and motor responses, comparing the two discrimination tasks (TD and SD) with the simple detection task (control) should indicate brain regions responsible for the analysis of somatosensory input (over and above primary sensory detection and response). More interestingly, direct comparison of the two discrimination tasks 
should highlight brain areas involved specifically in TD. Note that, unlike other temporal discrimination tasks (e.g., estimation of duration) that have been used to study the functional anatomy of temporal processes, our task does not rely on comparing the test stimulus with a previously presented reference stimulus. Instead, it requires the judgment of an absolute temporal aspect of the sensory input. This minimizes the involvement of memory processes.

\section{Materials and Methods Subjects}

Fourteen right-handed healthy volunteers (mean age, 28.9; SD, 5.1) were studied with approval from the Institute of Neurology and National Hospital for Neurology and Neurosurgery Joint Ethics Committee (University College of London Hospital Trust). Subjects also gave informed written consent after explanation of the experimental procedure. Before fMRI, subjects were first tested psychophysically to obtain individual discrimination thresholds.

\section{Stimuli}

Stimuli were delivered using an array of 16 pairs of electrodes embedded in a rubber sheet. The sheet was strapped around the left forearm and centered on the skin of the flexor middle third to enable stimulation of multiple sites with reference to anatomical cubital and radial landmarks. Eight pairs of electrodes were situated on the left and another eight pairs on the right (see Fig. $1 a$ for electrode spacing). The tactile stimuli were generated by a computer-controlled electric stimulator specifically built for this purpose. A personal computer (PC) specified the intensity, timing, and location of each stimulus. The computer also controlled the randomization procedure and collected the button press response events. During fMRI scanning, this PC was controlled by another PC, which controlled the visual display information and synchronized stimuli with the scanner (Cogent 2000 software; developed by the Cogent 2000 team, Functional Imaging Laboratory and Institute of Cognitive Neuroscience, Wellcome Department of Imaging Neuroscience). Stimulation comprised a pair of electric pulses of $0.2 \mathrm{msec}$ duration separated by various ISIs. The intensity of each pulse was set at twice the threshold intensity (determined separately for each electrode pair for each subject) and was felt as a brief, but not painful, tap.

\section{Psychophysical study}

\section{Discrimination tasks}

Temporal discrimination. A random series of paired stimuli with ISIs between 5 and $140 \mathrm{msec}$ was presented at a single location on the forearm. In each trial, subjects reported whether they felt one stimulus or a pair of distinct pulses. The response was a button press with the right hand on either the top or bottom key. Subjects were required to respond as fast as possible but maintain maximal accuracy. The stimulus-response association (one-two stimuli and top-bottom key) was counterbalanced across subjects.

Spatial discrimination. Pairs of stimuli (both at the same location) were presented randomly at each of the 16 electrode positions. In each trial, subjects discriminated the stimulus position as located to the left or right of an imaginary longitudinal midline through the flexor surface of the forearm. Subjects reported the perceived position with a button press, using the index finger of the right hand. Therefore, both the stimuli and responses were matched for the two discrimination tasks. To minimize stimulus-response compatibility effects, the two response buttons were placed in a "top versus bottom" configuration (i.e., orthogonal to the "left vs right" sensory judgment). The stimulus-response association (left-right stimulus and top-bottom key) was counterbalanced across subjects.

\section{Consistency index}

To index the influence of task difficulty associated with the presentation of stimuli at any given interval (TD) or any given location (SD), we introduced a measure of response consistency (consistency index) on the basis of the variability of the responses for each ISI (TD) or electrode position (SD). The following formula was used.

Consistency $=a b s(r 1-r 2) /(r 1+r 2)$, with $r 1$ and $r 2$ corresponding to the two types of responses (one and two for the temporal discrimination; left and right for the spatial discrimination). The consistency index equals unity if the subject always produces the same response for a given stimulus type. The index equals zero if the subject responds completely at chance ( $50 \%$ for each response type).

\section{fMRI study}

Experimental design. We used a factorial design that manipulated orthogonally the type of somatosensory discrimination (TD vs SD) and the consistency index of the task (high- vs low-response consistency).

Stimuli and tasks. For the fMRI scanning session, to reduce the total number of possible stimuli, we used essentially the same procedure as for the psychophysical study but selected only four electrode positions and four ISIs. Two were chosen in the "definite" regions (high consistency) and two in the "penumbra" regions for each subject (low consistency) (see Fig. $1 b, c$ ). The factorial combination of the four electrode positions by four time intervals gave a total of 16 possible stimulus types. Because only one stimulus attribute (either spatial or temporal) was judged at any time, these 16 stimulus types were collapsed to obtain eight experimental conditions: four conditions of spatial discrimination (two configurations with high-response consistency and two with low-response consistency), and four conditions of temporal discrimination (two configurations with high-response consistency and two with low-response consistency).

The same 16 stimulus types were used for TD, SD, and a control (C) task that involved simple detection of stimuli. For the control task, the subject was required to press a button as soon as a stimulus was detected, regardless of its location or ISI. Alternate buttons were pressed on successive presentations. Therefore, the control task used the same sensory stimulation as the two discrimination tasks, and the motor responses were well matched.

Scanning procedure. Each subject underwent three fMRI scanning runs. During each run, the two discrimination tasks (TD and SD) were presented in blocks of $40 \mathrm{sec}$. Each block consisted of eight trials, with the four trial types (two high-consistency and two low-consistency conditions) intermingled in a nonpredictable pseudorandom sequence. The inter-trial interval was $4 \mathrm{sec}$. The control blocks lasted for $20 \mathrm{sec}$ (four trials) and were presented between each discrimination block, leading to an alternating sequence: $4 \mathrm{C}-8 \mathrm{TD}-4 \mathrm{C}-8 \mathrm{SD}-4 \mathrm{C}-8 \mathrm{TD}-4 \mathrm{C}-8 \mathrm{SD}$ and so on. Over the three runs, each subject was presented with 144 trials for each discrimination condition (72 of which were associated with highconsistency responses, and 72 with low consistency) plus 144 trials for the simple detection control condition.

Throughout the experiment, a central fixation cross was displayed in front of the subject. The color of this fixation cross served to specify the current task. The association between the color of the fixation cross (red, green, or blue) and tactile task was arbitrary and was counterbalanced across subjects. For every change of task, an interval of 4 sec preceded the delivery of the first trial. This allowed for task switching. Subjects were required to maintain central fixation throughout each scanning run, and this was monitored with an eye-tracking device.

Data acquisition. Imaging was performed using a 2 tesla scanner (Siemens Vision, Erlagen, Germany) equipped with a head volume coil. The functional images sensitive to blood oxygenation level-dependent contrast were acquired by $\mathrm{T} 2{ }^{\star}$-weighted echoplanar imaging. Each functional image comprised 48 transverse slices [ $2 \mathrm{~mm}$ thickness; $1.8 \mathrm{~mm}$ gap; $64 \times 64,3 \times 3 \mathrm{~mm}$ pixels; echo time $(\mathrm{TE})=40 \mathrm{msec}$ ] covering the whole brain volume. A total of 190 sequential volumes was acquired per run with an effective repetition time (TR) of $3.65 \mathrm{sec}$. There were three scanning runs of $11.5 \mathrm{~min}$. To avoid systematic relationships between slice acquisition and stimulus presentation, the stimulus onset was randomly jittered with respect to the beginning of each volume acquisition.

fMRI analysis. We used Statistical Parametric Mapping (SPM) 99 software for image processing and analysis (http://www.fil.ion.ucl.ac.uk/ $\mathrm{spm} / \mathrm{spm} 99 . \mathrm{html}$ ). For each subject and run, the first four volumes were discarded to allow for $\mathrm{T} 1$ equilibration. The remaining 558 volumes were realigned to the first image, sinc-interpolated over time to correct for 


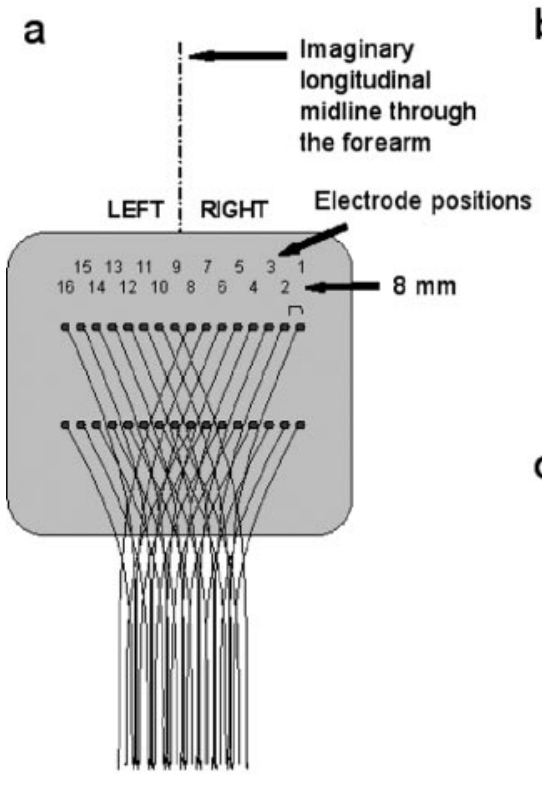

b

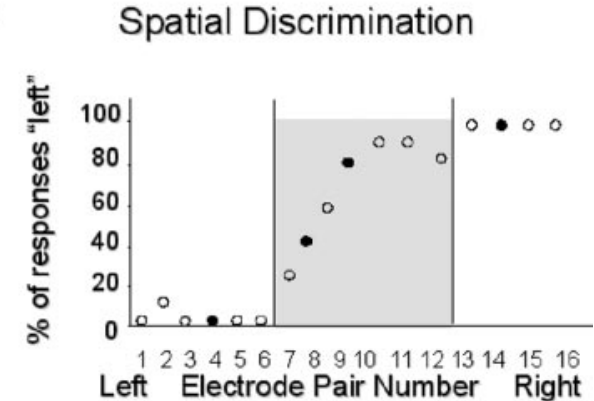

C

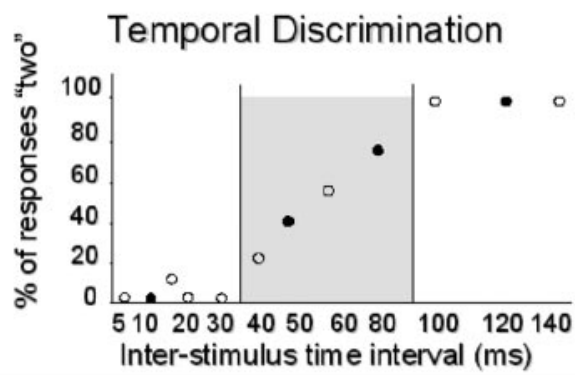

Figure 1. a, Schematic drawing of the electrode array, also showing the imaginary longitudinal midline used as a reference for the SD task. $b$, Behavioral performance of one representative subject for the SD task in the prescanning behavioral session. Behavioral performance of the same subject for the temporal discrimination task is shown. The filled circles in $b$ and c indicate electrode locations and time intervals chosen for fMRI scanning. Shaded areas correspond to the electrode positions and interstimulus intervals associated with inconsistent responses (i.e., penumbra).

phase advance during volume acquisition, and normalized to the Montreal Neurological Institute reference brain. The data were spatially smoothed with a Gaussian Kernel ( $10 \mathrm{~mm}$ full-width at half-maximum).

We used a two-level analysis procedure for statistical inference at a random effect level (i.e., all effects tested against the between-subjects variance). In the first level analysis (fixed effects), the time series of each subject was analyzed separately. For each subject, the eight event types (i.e., two types of discrimination by $2 \times 2$ levels of response consistency) were modeled using the appropriate stimulus function convoluted with a canonical hemodynamic response function (HRF). In addition, events occurring during the detection control task were randomly divided into two groups and again modeled using the standard HRF ( $\mathrm{df}=528)$.

For each single-subject model, we estimated contrasts of interest that were then assessed in the second level analysis (random effects) using a onesample $t$ test across the 14 subjects $(\mathrm{df}=13)$. Our study aimed to describe effects that are consistent across subjects. Inter-subject variability, in the context of our random effect analyses, will determine the size of SE.

We performed two main comparisons. First, we tested for the overall effect of discrimination (TD and SD) versus control (simple detection). This contrast highlights brain areas involved in both types of discrimination, regardless of electrode position and ISIs (i.e., common to definite and penumbra trials). Given the large number of voxel tested, we assessed statistical significance using the false discovery rate (FDR) approach, at $p$ corrected $=0.05$ (Genovese et al., 2002).

The second comparison addressed the main aim of the present study, which was to investigate neural systems specifically involved in one or the other tactile discrimination task. To assess this, we compared brain activity during TD versus SD, and vice versa. To test the hypothesis that peri-SMA areas, the basal ganglia, and inferior parietal cortex may participate in temporal discrimination, we selected the maximum significant voxels of activation in these areas in the first contrast (discrimination vs control) to obtain the centroid for a small volume correction (corrected threshold of $p=0.05$ ) in the contrast TD versus SD. It is important to note that these contrasts are orthogonal. In other words, the identification of areas involved in both types of discrimination compared with the control task does not bias toward differences in activation between temporal and spatial discrimination. Because there was no interaction between discrimination types and ISI-electrode configurations, we pooled across ISI-electrode configurations.

We report the anatomical location, $t, Z$, and $P$ values and Talairach coordinates for the voxel showing maximal statistical significance within activated clusters. For each activated area, we report the level of activity during each of the eight active conditions (four TD, four SD) compared with the two control conditions (see Figs. $3,4,5)$.

\section{Results \\ Behavioral data}

Before scanning, we estimated the individual thresholds for TD and SD. Fig 1 shows the results of this psychophysical procedure for one representative subject. Fig $1 b$ shows the percentage of left responses for each of the 16 possible stimulus positions. As stimulus location moves from left (electrode 16) to right (electrode 1), the percentage of left responses decreases. For electrodes around the midline (i.e., electrodes 7-10, for this subject), responses were less consistent (penumbra). Similar behavior was observed for the TD task. At short ISIs (i.e., $5-30 \mathrm{msec}$ in this subject), the subject consistently reported the perception of a single pulse, whereas at long ISIs ( $>100 \mathrm{msec})$, two pulses were reliably reported (definite). For ISIs ranging between 35 and $100 \mathrm{msec}$, the subject was less consistent (penumbra).

For scanning, we selected four electrode positions and four ISIs. Two were chosen in the definite domain and two in the penumbra regions for each subject (for this subject, see filled symbols in Fig. 1, $b$ and $c$ ). We quantified each subject's responses during fMRI in two ways: first by devising a consistency index on the basis of the variability of the responses (see Materials and Methods) and second by measuring reaction times.

The consistency index equals unity if the subject always produces the same response for a given stimulus type and zero if the subject responds completely at chance $(50 \%$ for each response type). The mean consistency indices across the 14 subjects are shown in Figure 2. A two-factor ANOVA [with discrimination task and stimulus configuration (ISIs or electrode position) as independent factors] revealed the expected main effect of stimulus configuration $(F=11.8 ; p=0.004)$, with higher consistency for ISIs-electrodes in the definite compared with penumbra regions. There was no effect of discrimination task $(F=0.26 ; p=$ $0.62)$ and no interaction between the factors $(F=0.65 ; p=0.43)$.

The mean reaction times were analyzed similarly. There was no significant main effect of discrimination task [mean (SEM): $\mathrm{TD}=919$ (19) $\mathrm{msec} ; \mathrm{SD}=880$ (31) msec; $F=3.898 ; p=0.096]$. In addition, there was neither an effect of stimulus configuration $(F=3.944 ; p=0.094)$ nor an interaction between the two factors $(F=3.231 ; p=0.123)$.

These data indicate that during fMRI scanning, the tasks were well matched and brain activity during the two discrimination tasks can be compared, without behavioral confound. Moreover, the lack of significant main effect of task in the reaction time data suggests that the subjects did not switch strategy between the two discrimination tasks (for example, by responding to the first 


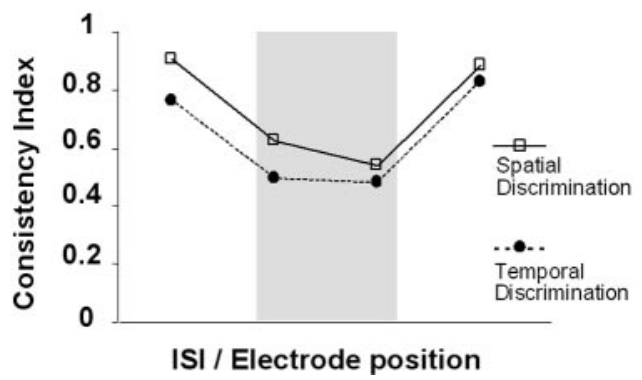

Figure 2. Grand mean of the consistency index for the 14 subjects, showing the behavioral performance during fMRI for SD (open squares, continuous line) and TD (filled circles, dotted line). On the $x$-axis are different ISI-electrode configurations, with short ISI (TD task) and leftward electrodes (SD task) plotted on the left of this graph (note that the exact ISI values and electrode position will vary for each subject, because these were determined individually). As expected, responses to electrode positions-intervals in the penumbra (gray shading) were less consistent than responses to limit-range conditions. Behaviorally, there was no significant effect of task or interaction between task and consistency.

stimulus during the SD task while waiting for the second in the TD task).

\section{fMRI results}

The analysis of fMRI data had two main aims: (1) to highlight brain areas involved in both types of tactile discrimination, over and above simple tactile detection and response, and (2) to investigate possible differences between the two types of tactile discrimination, with a specific interest in areas selectively activated during TD.

\section{Discrimination versus control}

Common areas activated by both types of discrimination versus control were located in cortical and subcortical regions, including the cerebellum (supplemental Table 1; available at www.jneurosci. org). Cortical activations were found in the right postcentral gyrus, inferior parietal lobule, middle and inferior frontal gyri, right insula in its anterior aspect, and right anterior cingulate. Subcortical clusters were observed in regions of the thalamus, head of caudate, and red nuclei-subthalamic nuclei (STN), whereas the cerebellar activation included the vermis and crus II bilaterally.

Figures 3 and 4 show the anatomical location and pattern of activity for several of these regions. They indicate the regions activated, regardless of discrimination task and stimulus condition. The parameter estimates in Figures 3 and 4 show activity during each discrimination task relative to activity during the control task. Zero represents the level of activity during the control task, and the bars represent deviations from control values in terms of the SE ( $t$ value).

None of the regions reported here showed a main effect of either discrimination task or stimulus condition, or an interaction between these two factors. Thus, the results suggest that a common network was activated for tactile discrimination, regardless of which attribute was being discriminated or response consistency.

\section{Main effect of discrimination}

To investigate the brain areas specifically involved in TD relative to $\mathrm{SD}$, we compared directly the activity during the two discrimination tasks. First, we compared TD versus SD. This showed increased activity in the medial aspect of the superior frontal gyrus in the region of pre-SMA, with a peak of maximal activity located in the right hemisphere (Fig. 5) (supplemental Table 2; available at www.jneurosci.org). The anterior cingulate was also activated, again with a peak located in the right hemisphere. Figure 5 shows the location and pattern of activity for the two clusters in the medial frontal lobe. The parameter estimates in Figure 5 illustrate that in these two areas, activity increased above control values only during the TD (first four bars from the left, in each plot). In these areas, there were no significant effects of stimulus configuration or interaction between discrimination and stimulus configuration. Thus, these areas appear to be engaged specifically during the analysis of temporal aspects of the somatosensory input, regardless of response consistency.

The reverse comparison (i.e., SD vs TD) revealed some activation of right superior parietal cortex and the precuneus, but these clusters did not survive our strict criteria for statistical significance $(x, y, z$ Talairach coordinates $=2,-60,64 ; t=6.06 ; z=$ 4.11; $p$ uncorrected $=0.0001 ; x, y, z$ Talairach coordinates $=0$, $-62,54 ; t=5.90 ; z=4.04 ; p$ uncorrected $=0.0001$, respectively) and are reported here only as trends. Finally, no brain region showed significant interaction between type of discrimination and stimulus configuration, suggesting that the regions involved in the analysis of specific somatosensory attributes (temporal or spatial) are not directly affected by the consistency of the percept associated with a particular attribute.

\section{Discussion}

We identified regions in the brain that respond selectively when discriminating specific attributes of a somatosensory stimulus as opposed to simply registering its presence. Furthermore, our results suggest that specialized networks are recruited for processing temporal attributes.

\section{Areas activated by both discrimination tasks}

When subjects were required to make judgments about the specific properties of a somatosensory stimulus, a number of cortical and subcortical regions was activated more than during a detection task. At the cortical level, these included the inferior parietal lobule, middle and inferior frontal gyri, anterior portion of the right insula, and right anterior cingulate gyrus. This pattern of activation can be mostly explained on the basis of the engagement of working memory centers (Maquet et al., 1996) and the frontoparietal network that allocates general attentional resources (Posner and Petersen, 1990; Klingberg et al., 1997). The posterior inferior frontal gyri have previously been found to be involved in selective attention to touch (Hagen et al., 2002).

Within somatosensory regions, we found modulation of the parietal operculum bilaterally, corresponding to the secondary somatosensory cortex (S2), and the inferior right postcentral gyrus contralateral to the stimulated arm. Presumably, the process of discrimination did not affect the primary somatosensory region of the forearm (S1), which should be located more dorsally. The finding of activation in S2 may relate to attentional modulation of the sensory input (Roland, 1981, 1982; Meyer et al., 1991; Drevets et al., 1995), an effect that in discrimination tasks is typically more pronounced in S2 than S1 (Burton et al., 1999). Similar effects have been observed at the single neuron level in primates. A change in synchronization and an $80 \%$ modulation of firing rate of neurons in S2 has been described in tactile shape discrimination tasks of varying difficulty (Steinmetz et al., 2000). During discrimination of a pair of vibrotactile stimuli, modulations in firing during the second stimulus were recorded in S2 but not in S1, indicating its participation in the discrimination processes (Romo et al., 2002).

At a subcortical level, the basal ganglia were activated during 


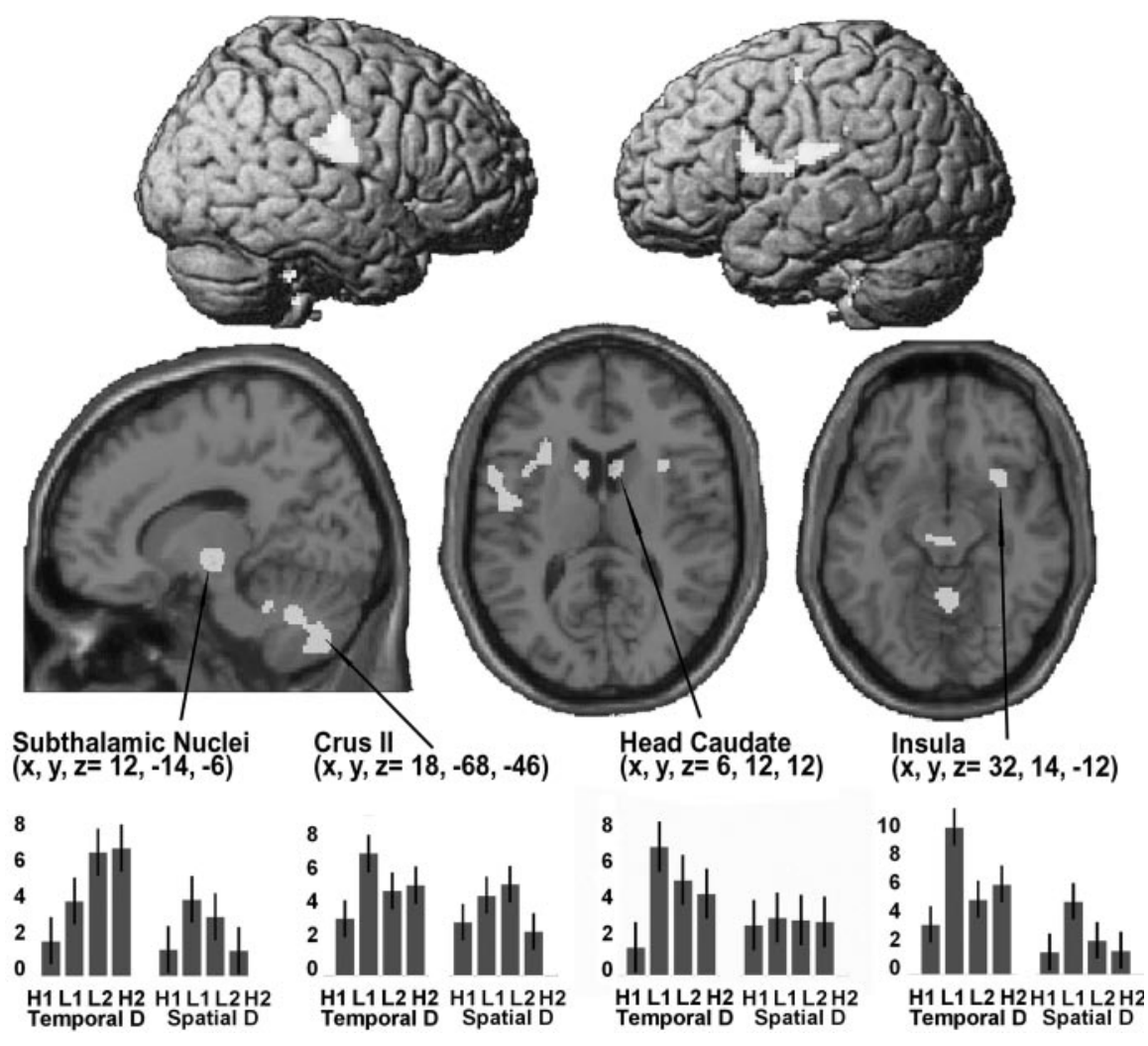

Figure 3. Top, Rendered view of the lateral surface of the left and right hemispheres, showing regions activating for both SD and TD, versus control. Bottom, Transverse sections on the canonical MR T1 template, showing anatomical details for the activations found in the head of caudate, right insula, and a sagittal section through subthalamic and cerebellar clusters (all activations are not visible in the surface-rendered projections). The plots show the pattern of activation for the four conditions of TD and four conditions of SD. Effect sizes represent the activation for each condition compared with baseline. The level of activity for each active condition corresponds to the contrast associated with the comparison of the active condition against baseline (using between-subject variability to calculate SE). As shown in the plots, all areas were activated above control, regardless of type of discrimination and consistency of response. SPM threshold $p$ FDR corrected $=0.05$. H/l, High/low-response consistency; R, right electrodes; 1, short ISIs; L, left electrodes; 2, long ISIs.

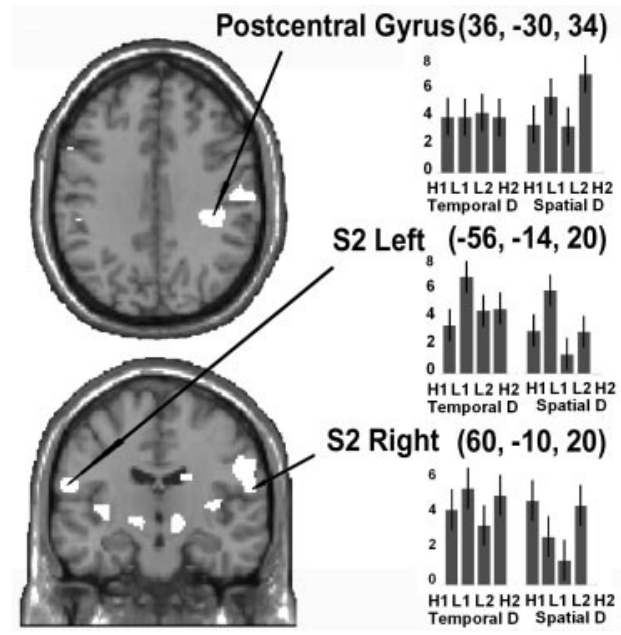

Figure 4. Coronal and transversal sections on the canonical MR T1 template, showing anatomical details for the activations found in postcentral gyrus and S2. The plots show the pattern of activation for the four conditions of TD and four conditions of SD. Effect sizes represent the activation for each condition compared with baseline. All areas were activated above control, regardless of type of discrimination and consistency of response. SPM threshold $p$ FDR corrected $=0.05$. H/l, High/low-response consistency; $R$, right electrodes; 1 , short ISIs; L, left electrodes; 2, long ISIs. both discrimination tasks. The areas included bilateral activation of the head of caudate, substantia Nigra, and STN. The latter are especially relevant, because they exert their effects on most components of the basal ganglia and their output centers (Smith et al., 1990; Parent and Hazrati, 1995; Middleton and Strick, 2002).

The strong activation of the cerebellum by both discrimination tasks, compared with stimuli detection with the same motor response, may reflect its role in optimizing perception of sensory inputs through interactions with the cerebral cortex (Bower, 1997; Middleton and Strick, 2001). The major bulk of cortical input to the cerebellum (via pons) comes from S1, followed by S2 and parietal areas 5 and 7 . The cerebellar clusters were located in the vermis, medial hemispheres lobule IV, VIIB, and VIIIA, and dentate nuclei symmetrically. These areas are in the neighborhood of the somatotopically distributed cerebellar tactile areas that are activated in roughness discrimination tasks (Parsons et al., 1997). The red nuclei, which were activated by TD and SD, also are activated in roughness discrimination tasks (Liu et al., 2000).

\section{Areas specific to \\ temporal discrimination}

No significant activation was found for the spatial task, which may have been attributable to the strict statistical criterion that was adopted. However, several areas of the brain were specifically activated during the temporal discrimination task when contrasted with the spatial task. These were located in the frontal cortex bilaterally, specifically pre-SMA and anterior cingulate gyri. For both tasks, we used identical stimuli sets and motor responses. Moreover, the behavioral results reflected similar reaction times and no difference in the consistency indices. Therefore, the differential activation pattern is likely to reflect the specific processing of temporal attributes of the somatosensory input.

Several imaging studies have shown participation of the SMA in other timing tasks such as visual or auditory rhythm discrimination (Schubotz et al., 2000) and duration discrimination (Pouthas et al., 2000; Rao et al., 2001; Ferrandez et al., 2003). This is close to but not coincident with the main area of activation during our temporal discrimination task, which was more anterior in the pre-SMA. This discrepancy may reflect the different timing processes or the different magnitude of time intervals investigated in these studies. The encoding of the duration of a time interval in the range of hundreds of milliseconds could differ from the detection of a gap between stimuli separated by a time interval in the tens of milliseconds range. It could also be related to differences in the sensory modality explored with somaesthetic inputs having the closest relationship to pre-SMA. However, with tactile stimuli, Macar et al. (2002) found participation of the SMA in a duration estimation and reproduction experiment. In this case, the subject was required to make an accurately timed motor 


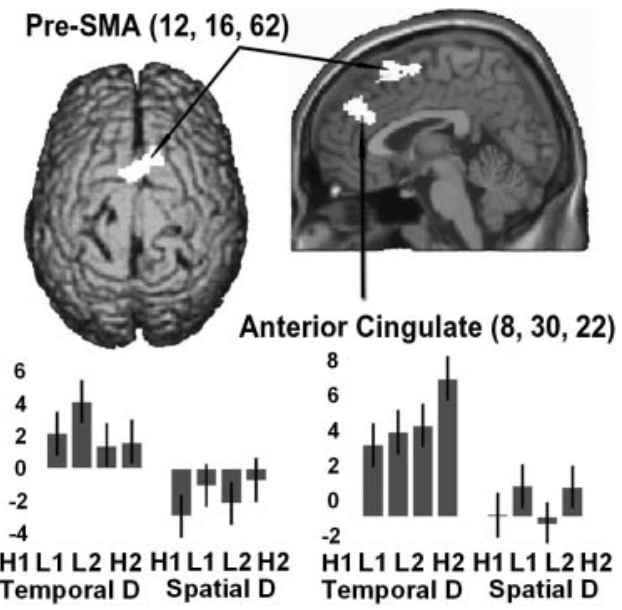

Figure 5. Activations observed for the direct comparison of TD versus SD rendered onto the canonical brain surface (top view) and onto a sagittal section close to the midline. The plots illustrate that only the TD task activated above control, and that activations occurred regardless of response consistency. SPM threshold $p$ small volume correction corrected $=0.05$. $\mathrm{H} / \mathrm{l}$, High/ low-response consistency; R, right electrodes; 1, short ISIs; L, left electrodes; 2, long ISIs.

response. It is possible that SMA involvement reflected preparation for this timed movement because SMA activation is known to relate to the temporal complexity of movement preparation (Grafton et al., 1992). Rao et al. (2001) found activation of prefrontal areas, which included pre-SMA and anterior cingulate, in duration and pitch discrimination tasks. However, in contrast to our results, these prefrontal areas were activated by both tasks. This difference needs additional investigation but it could relate to the fact that their tasks required more extensive encoding of stimuli and presumably a greater reliance on working memory. This extra processing might have masked the specific activation of pre-SMA for the temporal task.

Pre-SMA is considered part of the prefrontal cortex (Inase et al., 1999) because of its extensive connections with most prefrontal areas $(46,8 \mathrm{~A}, 9,10,11,12$, and 13$)$. It not only receives basal ganglia inputs via the VApc and VLcr but also cerebellar input via the VLcc and MDpl of the thalamus, and sends direct inputs to the striatum and subthalamic nuclei (Inase et al., 1996, 1999). Pre-SMA, strongly connected with SMA, is known to be involved in inter-hemispheric interactions (Liu et al., 2002) and in early phases of motor preparation and planning (Fujii et al., 2002). Others have suggested that pre-SMA plays an integrative role, for example, by relating external and motivational factors to movement initiation (Rizzolatti and Luppino, 2001) or by encoding differences between sensory stimuli and providing a link between perception and motor action (Hernandez et al., 2002). Pre-SMA has been shown to serve a timing function being activated by timed sequential movements (Ramnani and Passingham, 2001). Our findings are compatible with this idea of a timing function. One possibility is that pre-SMA, at least in the somatosensory realm, plays a role in the tagging of temporal attributes for subsequent processing by SMA and other brain regions. This hypothesis does not imply that pre-SMA plus anterior cingulate are the only areas involved in temporal processing. Several other regions were activated, but these were not specific for temporal discrimination [see Leon and Shadlen (2003) for related single cell findings in parietal cortex).

\section{Relationship to lesion studies}

Raised temporal discrimination thresholds have been reported in a variety of focal brain lesions (Lacruz et al., 1991). Of these, lesion sites in peri-SMA regions are of particular interest because they correspond to areas activated selectively in our temporal discrimination task. Other regions, especially the cerebellum, have been suggested to be involved in temporal processing, supported by duration discrimination studies using positron emission tomography (Jueptner et al., 1995), and the finding of abnormal performance in duration discrimination tasks in patients with cerebellar lesions (Ivry, 1996). Our study design illuminated the timing processes in a nonmotor task by presenting identical sets of stimuli with the same motor response but without reliance on a working memory representation. With this design, the cerebellum was not found to be involved in temporal discrimination specifically but showed activation in both temporal and spatial discrimination tasks. These results are in keeping with the idea that the cerebellum supports a more general role in processing of sensory information (Schmahmann and Sherman, 1998; Ohyama et al., 2003).

Focal lesions in the basal ganglia are also associated with raised temporal discrimination thresholds (Lacruz et al., 1991). Animal and human studies on the basal ganglia have suggested that the dopaminergic system plays a central timing role (Johnson and Harris-Warrick, 1990; Artieda et al., 1992; Pastor et al., 1992; Gibbon et al., 1997; Harrington et al., 1998; Meck and Benson, 2002; Nenadic et al., 2003). Patients with Parkinson's disease show increased temporal discrimination thresholds, with normal primary perception of sensory stimuli and normal somatosensory-evoked potentials-recovery curves after paired stimuli (Artieda et al., 1992). However, in the present study, basal ganglia activation occurred during discrimination, but it was common to both the spatial and temporal tasks.

Thus, our results predict that patients with dysfunctional basal ganglia and cerebellum should not only be impaired in temporal tasks but also in tasks involving spatial judgements (Gao et al., 1996; Weder et al., 1999; Bara-Jimenez et al., 2000; Molloy et al., 2003). Basal ganglia and cerebellar lesions could exert a more specific effect on timing function by disrupting their input to frontal cortex. Indeed, some models of temporal information processing consider that fronto-subcortical circuits linking the prefrontal cortical areas with the striatum and thalamus have a specific role in temporal processing (Gibbon et al., 1997). Thus, in line with our hypothesis, abnormal dopaminergic function may affect temporal discrimination by altering basal ganglia input to anterior cingulate and pre-SMA.

In conclusion, most of the areas that have been described previously as important for temporal processing were activated in both of our discrimination tasks. These include the cerebellum, basal ganglia, insulae, and regions of the inferior frontal cortex. However, pre-SMA and anterior cingulate were specifically activated during discrimination of temporal attributes of the somatosensory stimuli. These fronto-medial structures, directly and broadly connected with sensory and motor cortical areas of both hemispheres, as well as subcortical regions, are strategically placed for a pivotal role in temporal processing.

\section{References}

Artieda J, Pastor MA, Lacruz F, Obeso JA (1992) Temporal discrimination is abnormal in Parkinson's disease. Brain 115:199-210.

Bara-Jimenez W, Shelton P, Hallett M (2000) Spatial discrimination is abnormal in focal hand dystonia. Neurology 55:1869-1873.

Bower JM (1997) Control of sensory data acquisition. Int Rev Neurobiol 41:489-513.

Burton H, Abend NS, MacLeod AM, Sinclair RJ, Snyder AZ, Raichle ME (1999) Tactile attention tasks enhance activation in somatosensory re- 
gions of parietal cortex: a positron emission tomography study. Cereb Cortex 9:662-674.

Drevets WC, Burton H, Videen TO, Snyder AZ, Simpson Jr JR, Raichle ME (1995) Blood flow changes in human somatosensory cortex during anticipated stimulation. Nature 373:249-252.

Ferrandez AM, Hugueville L, Lehericy S, Poline JB, Marsault C, Pouthas V (2003) Basal ganglia and supplementary motor area subtend duration perception: an fMRI study. Neuroimage 19:1532-1544.

Fujii N, Mushiake H, Tanji J (2002) Distribution of eye- and armmovement-related neuronal activity in the SEF and in the SMA and preSMA of monkeys. J Neurophysiol 87:2158-2166.

Gao JH, Parsons LM, Bower JM, Xiong J, Li J, Fox PT (1996) Cerebellum implicated in sensory acquisition and discrimination rather than motor control. Science 272:545-547.

Genovese CR, Lazar NA, Nichols T (2002) Thresholding of statistical maps in functional neuroimaging using the false discovery rate. Neuroimage 15:870-878.

Gibbon J, Malapani C, Dale CL, Gallistel C (1997) Toward a neurobiology of temporal cognition: advances and challenges. Curr Opin Neurobiol 7:170-184

Grafton ST, Mazziotta JC, Woods RP, Phelps ME (1992) Human functional anatomy of visually guided finger movements. Brain 115:565-587.

Harrington DL, Haaland KY, Knight RT (1998) Cortical networks underlying mechanisms of time perception. J Neurosci 18:1085-1095.

Hagen MC, Zald DH, Thornton TA, Pardo JV (2002) Somatosensory processing in the human inferior prefrontal cortex. J Neurophysiol 88:1400-1406.

Hernandez A, Zainos A, Romo R (2002) Temporal evolution of a decisionmaking process in medial premotor cortex. Neuron Vol 33:959-972.

Inase M, Tokuno H, Nambu A, Akazawa T, Takada M (1996) Origin of thalamocortical projections to the presupplementary motor area (preSMA) in the macaque monkey. Neurosci Res 25:217-227.

Inase M, Tokuno H, Nambu A, Akazawa T, Takada M (1999) Corticostriatal and corticosubthalamic input zones from the presupplementary motor area in the macaque monkey: comparison with the input zones from the supplementary motor area. Brain Res 833:191-201.

Ivry RB (1996) The representation of temporal information in perception and motor control. Curr Opin Neurobiol 6:851-857.

Johnson BR, Harris-Warrick RM (1990) Aminergic modulation of graded synaptic transmission in the lobster stomatogastric ganglion. J Neurosci 10:2066-2076.

Jueptner M, Rijntjes M, Weiller C, Faiss JH, Timmann D, Mueller SP, Diener HC (1995) Localization of a cerebellar timing process using PET. Neurology 45:1540-1545.

Klingberg T, O'Sullivan BT, Roland PE (1997) Bilateral activation of frontoparietal networks by incrementing demand in a working memory task. Cereb Cortex 7:465-471.

Lacruz F, Artieda J, Pastor MA, Obeso JA (1991) The anatomical basis of somaesthetic temporal discrimination in humans. J Neurol Neurosurg Psychiatry 54:1077-1081.

Leon MI, Shadlen MN (2003) Representation of time by neurons in the posterior parietal cortex of the macaque neuron. 38:317-327.

Liu Y, Pu Y, Gao JH, Parsons LM, Xiong J, Liotti M, Bower JM, Fo PT (2000) The human red nucleus and lateral cerebellum in supporting roles for sensory information processing. Hum Brain Mapp 10:147-159.

Liu J, Morel A, Wannier T, Rouiller EM (2002) Origins of callosal projections to the supplementary motor area (SMA): a direct comparison between pre-SMA and SMA-proper in macaque monkeys. J Comp Neurol 443:71-85.

Macar F, Lejeune H, Bonnet M, Ferrara A, Pouthas V, Vidal F, Maquet P (2002) Activation of the supplementary motor area and of attentional networks during temporal processing. Exp Brain Res 142:475-485.

Maquet P, Lejeune H, Pouthas V, Bonnet M, Casini L, Macar F, TimsitBerthier M, Vidal F, Ferrara A, Degueldre C, Quaglia L, Delfiore G, Luxen A, Woods R, Mazziotta JC, Comar D (1996) Brain activation induced by estimation of duration: a PET study. Neuroimage 3:119-126.

Meck WH, Benson AM (2002) Dissecting the brain's internal clock: how frontal-striatal circuitry keeps time and shifts attention. Brain Cogn 48:195-211.
Meyer E, Ferguson SS, Zatorre RJ, Alivisatos B, Marrett S, Evans AC, Hakim AM (1991) Attention modulates somatosensory cerebral blood flow response to vibrotactile stimulation as measured by positron emission tomography. Ann Neurol 29:440-443.

Middleton FA, Strick PL (2001) Cerebellar projections to the prefrontal cortex of the primate. J Neurosci 21:700-712.

Middleton FA, Strick PL (2002) Basal ganglia "projections" to the prefrontal cortex of the primate. Cereb Cortex 12:926-935.

Molloy FM, Carr TD, Zeuner KE, Dambrosia JM, Hallett M (2003) Abnormalities of spatial discrimination in focal and generalized dystonia. Brain 126:1-8.

Nenadic I, Gaser C, Volz HP, Rammsayer T, Hager F, Sauer H (2003) Processing of temporal information and the basal ganglia: new evidence from fMRI. Exp Brain Res 148:238-246.

Nichelli P (1993) The neurophysiology of human temporal information processing. In: Handbook of neurophysiolgy, Vol IV (Boller F, Grafman J, eds), pp 339-369. Amsterdam: Elsevier.

Ohyama T, Nores WL, Murphy M, Mauk MD (2003) What the cerebellum computes. Trends Neurosci 26:222-227.

Parent A, Hazrati LN (1995) Functional anatomy of the basal ganglia. II. The place of subthalamic nucleus and external pallidum in basal ganglia circuitry. Brain Res Brain Res Rev 20:128-154.

Parsons LM, Bower JM, Gao JH, Xiong J, Li J, Fox PT (1997) Lateral cerebellar hemispheres actively support sensory acquisition and discrimination rather than motor control. Learn Mem 4:49-62.

Pastor MA, Artieda J, Jahanshahi M, Obeso JA (1992) Time estimation and reproduction is abnormal in Parkinson's disease. Brain 115:211-225.

Posner MI, Petersen SE (1990) The attention system of the human brain. Annu Rev Neurosci 13:25-42.

Pouthas V, Garnero L, Ferrandez AM, Renault B (2000) ERPs and PET analysis of time perception: spatial and temporal brain mapping during visual discrimination tasks. Hum Brain Mapp 10:49-60.

Ramnani N, Passingham RE (2001) Changes in the human brain during rhythm learning. J Cogn Neurosci 13:952-966.

Rao SM, Mayer AR, Harrington DL (2001) The evolution of brain activation during temporal processing. Nat Neurosci 4:317-323.

Rizzolatti G, Luppino G (2001) The cortical motor system. Neuron 31:889-901.

Roland PE (1981) Somatotopical tuning of postcentral gyrus during focal attention in man. A regional cerebral blood flow study. J Neurophysiol 46:744-754.

Roland PE (1982) Cortical regulation of selective attention in man. A regional cerebral blood flow study. J Neurophysiol 48:1059-1078.

Romo R, Hernández A, Zainos A, Lemus L, Brody CD (2002) Neuronal correlates of decision-making in secondary somatosensory cortex. Nat Neurosci 5:1217-1225.

Sanger TD, Tarsy D, Pascual-Leone A (2001) Abnormalities of spatial and temporal sensory discrimination in writer's cramp. Mov Disord 16:94-99.

Schmahmann JD, Sherman JC (1998) The cerebellar cognitive affective syndrome. Brain 121:561-579.

Schubotz RI, Friederici AD, von Cramon DY (2000) Time perception and motor timing: a common cortical and subcortical basis revealed by fMRI Neuroimage 11:1-12.

Smith Y, Hazrati LN, Parent A (1990) Efferent projections of the subthalamic nucleus in the squirrel monkey as studied by the PHA-L anterograde tracing method. J Comp Neurol 294:306-323.

Steinmetz PN, Roy A, Fitzgerald PJ, Hsiao SS, Johnson KO, Niebur E (2000) Attention modulates synchronized neuronal firing in primate somatosensory cortex. Nature 404:187-190.

Tinazzi M, Fiaschi A, Frasson E, Fiorio M, Cortese F, Aglioti SM (2002) Deficits of temporal discrimination in dystonia are independent from the spatial distance between the loci of tactile stimulation. Mov Disord 17:333-338.

Weder BJ, Leenders KL, Vontobel P, Nienhusmeier M, Keel A, Zaunbauer W, Vonesch T, Ludin HP (1999) Impaired somatosensory discrimination of shape in Parkinson's disease: association with caudate nucleus dopaminergic function. Hum Brain Mapp 8:1-12. 